Discrete Comput Geom 31:207-227 (2004)

DOI: $10.1007 /$ s00454-003-0729-3

\title{
Binary Space Partitions for Axis-Parallel Segments, Rectangles, and Hyperrectangles*
}

\author{
Adrian Dumitrescu, ${ }^{1}$ Joseph S. B. Mitchell, ${ }^{2}$ and Micha Sharir ${ }^{3}$ \\ ${ }^{1}$ Department of Electrical Engineering and Computer Science, \\ University of Wisconsin-Milwaukee, \\ Milwaukee, WI 53211, USA \\ ad@cs.uwm.edu \\ ${ }^{2}$ Department of Applied Mathematics and Statistics, Stony Brook University, \\ Stony Brook, NY 11794, USA \\ jsbm@ams.sunysb.edu \\ ${ }^{3}$ School of Computer Science, Tel Aviv University, \\ Tel Aviv 69978, Israel \\ michas@tau.ac.il \\ and \\ Courant Institute of Mathematical Sciences, New York University, \\ New York, NY 10012, USA
}

\begin{abstract}
We provide a variety of new upper and lower bounds and simpler proof techniques for the efficient construction of binary space partitions (BSPs) of axis-parallel rectangles of various dimensions. (a) We construct a set of $n$ disjoint axis-parallel segments in the plane such that any binary space auto-partition has size at least $2 n-o(n)$, almost matching an upper bound of d'Amore and Franciosa. (b) We establish a similar lower bound of $7 n / 3-o(n)$ for disjoint rectangles in the plane. (c) We simplify and improve BSP constructions of Paterson and Yao for disjoint segments in $\mathbb{R}^{d}$ and disjoint rectangles in $\mathbb{R}^{3}$. (d) We derive a worst-case bound of $\Theta\left(n^{5 / 3}\right)$ for the size of BSPs of disjoint 2-rectangles in 4-
\end{abstract}

* A preliminary version of this paper appears in Proc. 17th Annual ACM Symposium on Computational Geometry, June 3-5, 2001, Medford, MA. Work by Joe Mitchell has been partially supported by the U.S.-Israeli Binational Science Foundation, HRL Laboratories, Metron Aviation, National Science Foundation (CCR9732221, CCR-0098172), NASA Ames Research Center (NAG2-1325), Northrop-Grumman Corporation, Sandia National Labs, Seagull Technology, and Sun Microsystems. Work by Micha Sharir has been supported by NSF Grant CCR-97-32101, by a grant from the U.S.-Israeli Binational Science Foundation, by a grant from the Israeli Academy of Sciences for a Center of Excellence in Geometric Computing at Tel Aviv University, by the ESPRIT IV LTR Project No. 21957 (CGAL), and by the Hermann Minkowski-MINERVA Center for Geometry at Tel Aviv University. Part of the work on this paper was done when Joe Mitchell visited Tel Aviv University during July-August 2000. 
space. (e) For disjoint $k$-rectangles in $d$-space, we prove the worst-case bound $\Theta\left(n^{d /(d-k)}\right)$, for any $k<d / 2$; this bound holds for all $k<d$ if the rectangles are allowed to intersect.

\section{Introduction}

Let $S$ be a collection of $n$ objects in $\mathbb{R}^{d}$. A binary space partition (or $B S P$ for short) for $S$ is a recursively defined convex subdivision of space obtained by cutting space into two open regions $C$ and $C^{\prime}$ by a hyperplane and recursively constructing BSPs for the sets $\{s \cap C \mid s \in S\}$ within $C$ and $\left\{s \cap C^{\prime} \mid s \in S\right\}$ within $C^{\prime}$. The process terminates when each (open) cell of the BSP intersects at most one object of $S$. The input objects are usually assumed to be pairwise disjoint; however, the definition of a BSP we have given is applicable to any set of objects in $\mathbb{R}^{d}$ for which no pair of objects intersect in a $d$-dimensional set. In particular, the definition applies to sets of objects $S$ that are not $d$-dimensional. The definition also applies to sets of curved objects; however, the number of cells of the BSP will depend on the complexity of polyhedral separators between pairs of objects, and a BSP may not exist if the curved objects are allowed to intersect. Throughout this paper, objects will be assumed pairwise disjoint unless specified otherwise.

When talking about BSPs there are three different types: arbitrary, axis-parallel, and auto-partitions. In an axis-parallel partition, each cutting hyperplane is of the form $x_{i}=c$. If $S$ is a set of polyhedral objects in $\mathbb{R}^{d}$, and the cuts are always made along supporting hyperplanes of objects in the set, the resulting partition is called an autopartition.

Binary space partitions were introduced by the computer graphics community [6]-[8] and have numerous applications for rendering, ray shooting and tracing, solid modeling, rectangle tiling, etc. See [5] and [13].

Ideally, a BSP should partition space so that each object lies entirely in a single cell or in a cutting hyperplane; see [3]. However, in most cases this is impossible; cutting space (and objects) with a hyperplane may create fragments of objects that either lie in one of the two open halfspaces bounded by the hyperplane or are contained in the hyperplane. The size of a BSP for a set of $k$-dimensional objects in $\mathbb{R}^{d}$ is the number of $k$-dimensional fragments of objects of $S$ that it produces; in the ideal case, the size is $n$. We remark that one often defines the size of a BSP as the number of convex regions of $\mathbb{R}^{d}$ in the decomposition. Here, we opt for the definition in terms of the number of object fragments; this choice makes some of the analysis cleaner, particularly in the case of BSPs of low-dimensional hyperrectangles within higher-dimensional spaces. See also additional comments at the end of this Introduction.

One of the major research directions in this area is to construct BSPs of small size. Paterson and Yao [9], [10] proved that for a set of $n$ line segments in the plane there exists a BSP of size $O(n \log n)$, and when the segments are rectilinear (i.e., parallel to the coordinate axes), there exists a BSP of size at most $3 n$. They also conjectured that any set of $n$ line segments admits a BSP of size $O(n)$. Only very recently, a lower bound of $\Omega(n(\log n / \log \log n))$, in the worst case, was obtained by C. Tóth [11]. For both the general case and the axis-parallel case, BSPs with the above size bounds can be computed in $O(n \log n)$ time. Linear size bounds for BSPs were obtained for sets of fat 
objects, for sets of line segments having a bounded constant ratio between the lengths of the longest and the shortest segment, for sets of homothetic objects, and for sets of disjoint segments touching a common convex boundary [2]. A linear bound for segments in the plane with a fixed number of orientations was recently obtained by C. Tóth [12].

The bound $3 n$ of Paterson and Yao [10] on the size of a BSP for $n$ axis-parallel line segments was (implicitly) improved by d'Amore and Franciosa [1] to $2 n$. They explicitly obtained an upper bound of $4 n$ for axis-parallel rectangles in the plane. By specializing their analysis to the case of segments, one obtains the bound $2 n$ (or rather $2 n-1$, with an obvious optimization). Berman et al. [4] used the same approach, accompanied by an improved charging scheme, to reduce the upper bound for the case of axis-parallel rectangles to $3 n$.

In this paper we consider the case in which $S$ is a collection of axis-parallel ${ }^{1}$ hyperrectangles of various dimensions in $\mathbb{R}^{d}$ (or $k$-rectangles ${ }^{2}$ for various $k$ ). While we do consider some cases in which the hyperrectangles are allowed to intersect, we assume from now on, unless otherwise specified, that they are pairwise disjoint. The simplest such case is that of line segments (or rectangles) in the plane, mentioned above. In higher dimensions, Paterson and Yao [10] show that if $S$ is a set of $n$ (axis-parallel) line segments in $\mathbb{R}^{d}$, then $S$ admits a BSP of size $O\left(n^{d /(d-1)}\right)$, and that this bound is tight in the worst case. They also consider the case of rectangles in $\mathbb{R}^{3}$ and show that the same bound, $O\left(n^{3 / 2}\right)$, can also be obtained for rectangles (and also for boxes). They leave as an open problem obtaining sharp bounds for the size of BSPs for higher-dimensional hyperrectangles in dimension 4 and higher.

In this paper we improve and simplify the analysis leading to the bounds obtained by Paterson and Yao [10] and derive new bounds for the cases left open in that paper. Specifically, we first consider the case of axis-parallel line segments in the plane. We show, in Section 2, that there exist collections of $n$ (axis-parallel) line segments in the plane for which any rectilinear BSP has size at least $2 n-o(n)$, thus showing that the upper bound of d'Amore and Franciosa [1] is tight in the worst case apart for lower-order terms. This answers an open problem posed by S. Smorodinsky at EuroCG '2000. We also give an improved lower bound for the case of rectangles in the plane, showing that in the worst case a BSP must have size at least $7 n / 3-o(n)$.

We then consider the case of axis-parallel line segments in $\mathbb{R}^{d}$, and obtain, in Section 3 , a very simple alternative construction of BSPs of size $O\left(n^{d /(d-1)}\right)$. In addition to being simpler, the constants of proportionality that our method yields appear to be considerably smaller than those produced by the technique of Paterson and Yao [10]. We also consider the case of rectangles in $\mathbb{R}^{3}$, and again present an alternative simple construction of a BSP of size $O\left(n^{3 / 2}\right)$.

Next, in Sections 4 and 5, we consider higher-dimensional cases. We provide the first nontrivial bounds on the worst-case size of BSPs for hyperrectangles of dimension greater than 1 in higher dimensions, showing that, for any $k<d / 2$, there exist BSPs of

\footnotetext{
${ }^{1}$ From now on, we freely drop the adjective "axis-parallel"; in all cases the segments, rectangles, or hyperrectangles we consider are axis-parallel.

${ }^{2}$ A $k$-rectangle, or a $k$-dimensional hyperrectangle, is the Minkowski sum of $k$ mutually orthogonal axisparallel segments. The endpoints of such segments define the extents of a hyperrectangle in the corresponding dimensions.
} 
Table 1. Summary of our bounds.*

\begin{tabular}{lcll}
\hline$d$ & $k$ & \multicolumn{1}{c}{ Upper bound } & \multicolumn{1}{c}{ Lower bound } \\
\hline 2 & 1 & $2 n-1(\dagger[1])$ & $2 n-o(n)$ \\
2 & 2 & $3 n(\dagger[4])$ & $7 n / 3-o(n)$ \\
$d$ & 1 & $O\left(n^{d /(d-1)}\right)(*[10])$ & $\Omega\left(n^{d /(d-1)}\right)(*[10])$ \\
3 & 2 & $O\left(n^{3 / 2}\right)(*[10])$ & $\Omega\left(n^{3 / 2}\right)(*[10])$ \\
$d$ & $k<d / 2$ & $O\left(n^{d /(d-k)}\right)$ & $\Omega\left(n^{d /(d-k)}\right)$ \\
$d$ & $k \leq d-1$, & $O\left(n^{d /(d-k)}\right)$ & $\Omega\left(n^{d /(d-k)}\right)$ \\
& intersecting & & \\
4 & 2 & $O\left(n^{5 / 3}\right)$ & $\Omega\left(n^{5 / 3}\right)$ \\
\hline
\end{tabular}

*A bound tagged by $(\dagger)$ indicates a known bound, given for reference. A bound tagged by $(*)$ indicates a known bound, re-derived here with a simpler proof. All objects are pairwise disjoint unless indicated otherwise.

size $O\left(n^{d /(d-k)}\right)$ for any set of $n k$-rectangles in $d$ dimensions, and that this bound is tight in the worst case. This bound subsumes the bound $\Theta\left(n^{d /(d-1)}\right)$ for segments, mentioned above. In fact, the $\Theta\left(n^{d /(d-k)}\right)$ bound holds for any $k \leq d-1$ if the rectangles are allowed to intersect.

The next simplest case that is not covered by the results reported so far is the case $k=2$ and $d=4$. We show that a BSP of size $O\left(n^{5 / 3}\right)$ exists for any set of $n$ disjoint 2-rectangles in $\mathbb{R}^{4}$, improving the bound $O\left(n^{2}\right)$ that follows from the general bounds (for possibly intersecting rectangles) stated in the preceding paragraph. We also have a matching lower bound of $\Omega\left(n^{5 / 3}\right)$, showing that our upper bound is tight in the worst case.

Our results are summarized in Table 1.

We make one final remark regarding the definition of BSP and how it applies to any set of hyperrectangles that are not in general position ${ }^{3}$ in higher dimensions. One can modify the definition of BSP in order to require that the intersection of objects with any cutting hyperplane are recursively decomposed with a BSP of one lower dimension. This modification is useful in some applications; e.g., see [14]. It does lead to different complexity bounds on the size of the resulting decomposition, since, for instance, a set of $n$ line segments that lie in a common hyperplane $h$ in $\mathbb{R}^{4}$ requires only a single cutting hyperplane $(h)$ for a BSP by our definition, resulting in two cells and size $n$ (since there are $n$ object fragments). However, the $n$ segments may form a configuration in the three-dimensional space of the hyperplane $h$ such that the decomposition of $h$ by a three-dimensional BSP of the segments requires size $\Omega\left(n^{3 / 2}\right)$ [10, Section 6]. In this paper we use the strict definition of BSP made earlier (and introduced by Fuchs et al. [8]), not requiring that lower-dimensional subconfigurations be recursively decomposed. If the input objects are in general position, our bounds hold for either definition; in case the input is not in general position, though, our bounds do not hold for the other more refined definition.

\footnotetext{
${ }^{3}$ Here, a set of hyperrectangles is said to be in general position if for each $i$ the $x_{i}$-coordinates that define the extents of the hyperrectangles are all distinct.
} 


\section{Segments and Rectangles in Two Dimensions}

We start with the case in which $S$ is a set of $n$ axis-parallel pairwise disjoint line segments in the plane. By applying the method of d'Amore and Franciosa [1], originally designed for the case of rectangles (based on a minor variant of Paterson and Yao's method [10]), we readily obtain the following result for line segments:

Theorem 2.1. A set of $n$ axis-parallel and pairwise disjoint line segments in the plane admits a binary space auto-partition of size at most $2 n-1$. This BSP can be computed using $O(n \log n)$ time and space and has the additional property that no input segment is cut more than once.

We turn our attention now to establishing the tightness of the upper bound. The best construction known prior to this work is a cycle of thickness $n / 4$, shown in Fig. 1(a) (for $n=20$ ), which requires a BSP of size at least $5 n / 4$.

Theorem 2.2. There exists a set $S$ of $n$ disjoint axis-parallel line segments with the property that any axis-parallel binary space partition of S has size at least $2 n-O\left(n^{2 / 3}\right)$.

Proof. We give the proof for the case of auto-partitions, and note that it extends to axis-parallel partitions in a straightforward way. Since we deal with auto-partitions, all cuts are axis-parallel and along supporting lines of the segments. If $s, t \in S$, we say that $s$ cuts $t$ if by extending the supporting line of $s$ (within the cell of the current BSP containing $s$ and $t$ ), the segment $t$ is the first that is cut in two parts. Any segment can cut at most two other segments (one on each side of $s$ ). This defines a directed simple graph $G=(S, E)$ with vertex set $S$, and edge set $E$ given by the (asymmetric) cut relation. A cycle in $G$ is minimal if no proper subset of it is a cycle.

In general, the size of the BSP is $n+c_{n}$, where $c_{n}$ denotes the number of cuts that are made during the partitioning process. Since $c_{n} \geq n / 4$ in the example of Fig. 1(a), it yields the lower bound $n+n / 4=5 n / 4$.

Our construction for the lower bound is based on a grid-like configuration of segments, placed within some fixed axis-parallel square $R$, as illustrated in Fig. 1(b), (c). We parameterize it by the number of anchored horizontal segments on the left side of $R$, and the number of anchored vertical segments on the bottom side of $R$ : if these numbers are $k$ and $l$ respectively, we have a $k \times l$ simple grid (or a $k \times l 1$-grid); see Fig. 1(b). We restrict our attention to the set $\mathcal{C}$ of minimal cycles of length 4 associated with the (large and small) cells in the grid. It is easy to see that a BSP is obtained only when all cycles in the set $\mathcal{C}$ have been cut; that is, at least one of the segments participating in each cycle has been cut, and the new portions of these segments no longer form a cycle.

First, consider a $k \times k 1$-grid, to obtain a lower bound on $c_{n}$. The number of cells is $e=k^{2}+(k-1)^{2}, k^{2}$ of which are small cells, and the total number of segments is $n=2 k(k+1)$. Each small cell is a cycle and requires at least one cut by itself. We have $c_{n} \geq k^{2}=n / 2-o(n)$, from which we get a lower bound of $1.5 n-o(n)$ on the size of any BSP for this configuration. 


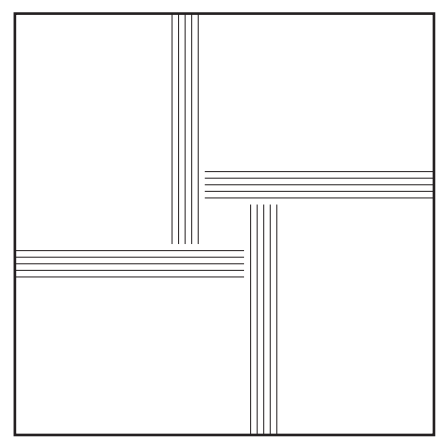

(a)

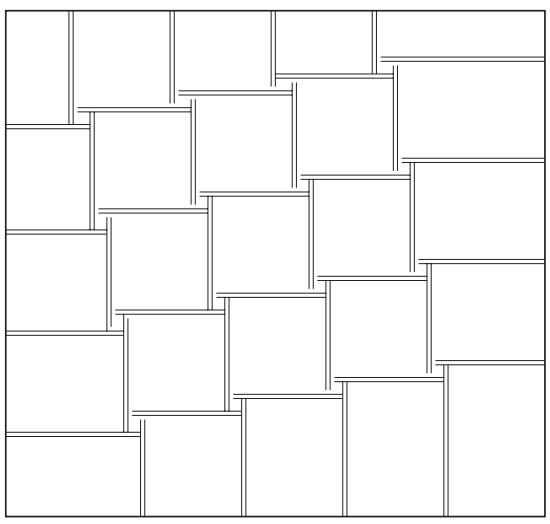

(c)

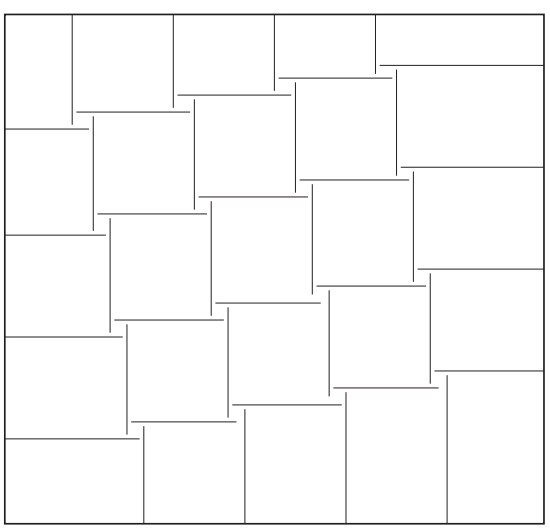

(b)

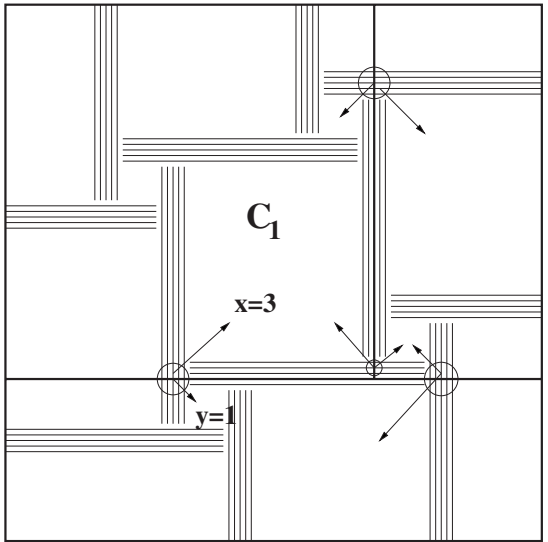

(d)

Fig. 1. (a) A cycle configuration of thickness $w=5$. (b) A $4 \times 41$-grid. (c) A $4 \times 42$-grid (double grid). (d) Charging scheme in a 5-grid.

In order to improve this lower bound, we consider a $k \times k$ m-grid, in which each single segment of the simple $k \times k 1$-grid is replaced by a set of $m$ parallel segments of equal length. A 2-grid is shown in Fig. 1(c), and a portion of a 5-grid is shown in Fig. 1(d). For a $k \times k m$-grid, there are $n=2 m k(k+1)$ segments and $e=k^{2}+(k-1)^{2}$ cells.

We claim that $c_{n} \geq\left(m-\frac{1}{2}\right) e$; selecting $m=k$ implies a lower bound of $2 n-O\left(n^{2 / 3}\right)$ on the size of any auto-partition BSP for this configuration, since

$$
\frac{c_{n}}{n}=\frac{\left(2 k^{2}-2 k+1\right)(2 k-1)}{4 k^{2}(k+1)}=1-O\left(\frac{1}{k}\right)=1-O\left(n^{-1 / 3}\right),
$$

using the fact that $n=\Theta\left(k^{3}\right)$.

For a given cell $C$, denote by $A(C)$ the set of $4 m$ segments associated with $C$; namely, $A(C)$ consists of $m$ segments on each of the four sides of $C$. We say that cell $C$ is touched by a cutting line $l$ if either (i) $l$ intersects the interior of the cell, viewed as a convex 
region (square), or (ii) $l$ is one of the supporting lines of the $4 m$ segments associated with $C$. Case (i) defines a middle cut, case (ii) defines a boundary cut.

The (current) thickness of $C$, denoted $w(C)$, is the minimum number of segments that one needs to cut so that all cycles determined by $A(C)$ are eliminated. We observe an equivalent characterization of the (current) thickness: it is the minimum number of segments that one needs to cut, such that $w(C)$ is strictly decreased in one cutting step. In the example of Fig. 1(d), the thickness of the cell $C_{1}$ after the first horizontal cut is 3 , and one needs to cut at least three segments to reduce it. After the second vertical cut, the thickness becomes 2 . The thickness of each cell is decreased during the cutting process, from $m$ to 0 , through one or more cuts.

To obtain a lower bound for $c_{n}$, we distribute the cost of each cutting line drawn in the BSP construction among the cells that are cut. In this manner, cells accumulate charges made by the cuts of the BSP, and our scheme will guarantee that by the end of the process each cell will be charged at least $m-\frac{1}{2}$, which will thus imply the asserted lower bound for $c_{n}$. Our charging scheme maintains the invariant that if a cell has been touched, and its current thickness is $w(0 \leq w \leq m-1)$, it has been charged at least $m-w-\frac{1}{2}$ units. An illustration of this charging scheme on the first few cuts in a 2-grid appears in Fig. 2.

Let us examine the first cut that touches a fixed cell $C_{1}$. The number of locally cut segments in $A\left(C_{1}\right)$ is $c=m$. If we have a middle cut, we charge $C_{1}$ with the entire cost $m$. The invariant is clearly maintained for $C_{1}$ (the cell is charged with at least $m-\frac{1}{2}$ units). If we have a boundary cut, the local cost of the cut (namely, $m$ ) is distributed to cell $C_{1}$ and its adjacent cell $C_{2}$ (with respect to the common boundary and the segments locally cut) as illustrated in Fig. 1(d): $m-x-\frac{1}{2}$ to cell $C_{1}$ and $m-y-\frac{1}{2}$ to cell $C_{2}$. Here $x$ (resp. $y$ ) is the number of segments in $A\left(C_{1}\right)$ on the same side with $C_{1}$ (resp. separated from $C_{1}$ ) with respect to the cutting line. The total charge is $\left(m-x-\frac{1}{2}\right)+\left(m-y-\frac{1}{2}\right)=m$, since $x+y+1=m$. Thus the invariant holds for $C_{1}$ after the first cut that touches $C_{1}$ is made.

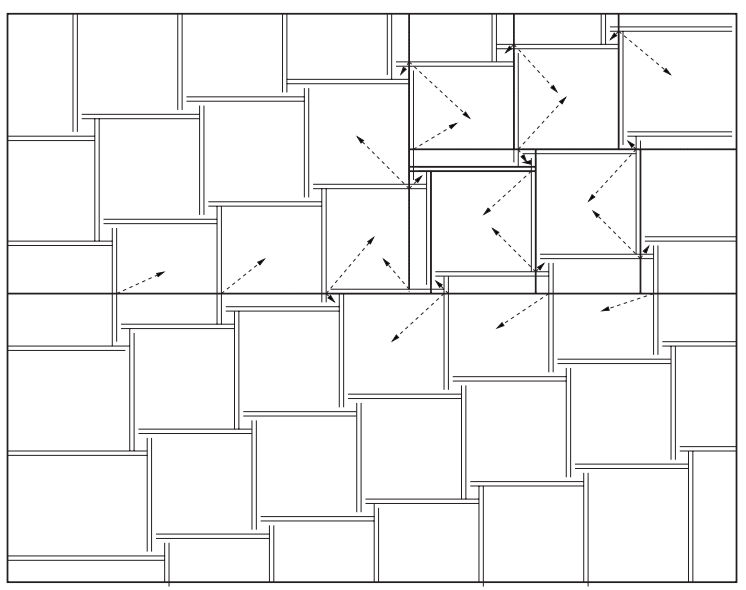

Fig. 2. Charging scheme in a $5 \times 62$-grid. 
For any subsequent cut, let $c$ be the number of locally cut segments in $A\left(C_{1}\right)$, and let $w_{1}$ and $w_{1}^{\prime}$ denote the thickness of $C_{1}$ before and after the cut, respectively. We distinguish two cases:

Case 1: The cut does not reduce the thickness of the adjacent cell $C_{2}$. If the cut does not reduce the current thickness of $C_{1}$ (i.e., $w_{1}=w_{1}^{\prime}$ ), we assign the whole local cost of the cut to $C_{1}$, clearly maintaining the invariant. If it does reduce $w_{1}$ (i.e., $w_{1}^{\prime}<w_{1}$ ), we only have to ensure that $c \geq w_{1}-w_{1}^{\prime}$ to maintain the invariant. However, this is clear, since $c \geq w_{1}$ from our second (equivalent) characterization of thickness.

Case 2: The cut reduces the thickness $w_{2}$ of the adjacent cell $C_{2}$ (as well as the thickness $w_{1}$ of $C_{1}$, otherwise there is no need to assign any cost to $C_{1}$ ). We only have to ensure that $c \geq\left(w_{1}-w_{1}^{\prime}\right)+\left(w_{2}-w_{2}^{\prime}\right)$ (where $w_{2}^{\prime}$ is the thickness of $C_{2}$ after the cut) to maintain the invariant. Since the thickness for both $C_{1}$ and $C_{2}$ is reduced, we must be looking at a common boundary cut of $C_{1}$ and $C_{2}$ and then $w_{1}^{\prime}+w_{2}^{\prime}=m-1$ (the cutting line is along a segment of $\left.A\left(C_{1}\right) \cap A\left(C_{2}\right)\right)$. It is thus enough to show that $c+m-1 \geq w_{1}+w_{2}$, which follows from the inequalities $c \geq w_{2}$ and $m-1 \geq w_{1}$. The first is implied by our equivalent characterization of thickness (more generally, $c \geq \max \left(w_{1}, w_{2}\right)$ ), and the second holds since this is not the first cut for $C_{1}$.

After the partitioning process ends, each cell has thickness 0 , and its charge is at least $m-\frac{1}{2}$, which, as noted, completes the proof of the theorem.

We turn now to the case in which $S$ is a set of $n$ disjoint axis-parallel rectangles. The best known upper bound on the size of the BSP is $3 n$, given in the recent paper of Berman et al. [4], improving the prior bound of $4 n$ [1]. Our construction for line segments gives immediately a lower bound of $2 n-o(n)$ (a similar bound was also obtained independently by Berman et al. [4]; however, their bound applies only for rectangles, not for segments). We are able to show an even better lower bound for rectangles:

Theorem 2.3. There exists a set $S$ of $n$ disjoint axis-parallel rectangles (in fact, of $n$ unit squares, as in Fig. 3) with the property that any axis-parallel binary space partition of $S$ has size at least $7 n / 3-O(\sqrt{n})$.

Proof. Consider a $k \times k$ square configuration (with $n=k^{2}$ unit squares) with the pattern shown in Fig. 3. We have $s=(k-1)^{2}$ "junctions," corresponding to the small cells in the grid configuration (of size $\epsilon>0$ ), at each of which four squares are "meeting." Consider any orthogonal cut in the BSP, having (physical) length $l$. We distinguish three types of parts in our cut: (i) the border part, if any, of length $l_{b} \geq 0$ lies on a side of a square or along the sides of two adjacent squares (adjacency in the grid refers to the $\mathrm{N}$, $\mathrm{S}, \mathrm{E}, \mathrm{W}$ squares only); (ii) the aligned part, if any, of length $l_{a} \geq 0$ measures the at most two parts of length $\leq 1$ adjacent to the border part; (iii) the middle part, if any, of length $l_{m} \geq 0$ encompasses the rest of the length.

We have $l=l_{b}+l_{a}+l_{m}$, where $l_{b} \leq 1+\epsilon$ and $l_{a} \leq 2$. As before, let $c_{n}$ denote the number of cuts (additional number of object parts in the BSP); then the size of the BSP is $n+c_{n}$. It is easy to see that each of the $(k-1)^{2}$ junctions will create at least one cut (which materializes along an adjacent aligned portion of the cut). This gives us a lower bound of $2 n-o(n)$ on the size of the BSP. To account for more, we will prove a lower 


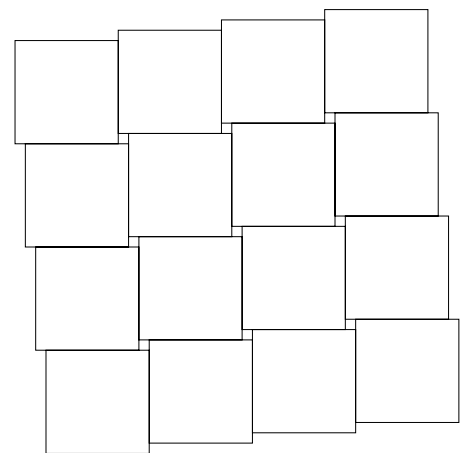

Fig. 3. A set of disjoint unit squares used in the lower bound of Theorem 2.3.

bound on the number of additional parts generated by middle portions of the cuts: unless a rectangular subcell $R$ obtained during the BSP construction has one "short" side, any cut of $R$ will generate additional parts due to the middle part component, which are unaccounted for by the junctions inside the rectangle. For $i, j \geq 0$, denote by $T(i, j)$ the minimum number of additional "middle" parts in the BSP attributed to the middle portions of the cut, obtained when a rectangle containing a complete $i \times j$ array of interior junctions is cut by a line. It is easy to see that $T(3,3) \geq 1$ (a $3 \times 3$ array of interior junctions is illustrated in Fig. 3). A lower bound on the size of a BSP (with $n=k^{2}$ ) for a $k \times k$ square configuration is

$$
n+c_{n}=k^{2}+(k-1)^{2}+T(k-1, k-1) .
$$

We claim that $T(i, j) \geq(i-2)(j-2) / 3$ for any $i, j \geq 3$. This claim implies that

$$
n+c_{n}=k^{2}+(k-1)^{2}+\frac{(k-3)^{2}}{3}=\frac{7}{3} n-O(\sqrt{n})
$$

completing the proof of the theorem. We prove our claim by induction on $i+j$ as follows.

The basis $i=j=3$ holds by the above observation. Without loss of generality assume the orthogonal cut splits the rectangle containing the $i \times j$ array of junctions into two subrectangles containing $i \times j_{1}$ and $i \times j_{2}$ arrays of junctions, where $j-1 \leq j_{1}+j_{2} \leq j$ and $j_{1} \leq j_{2}$. Then

$$
T(i, j) \geq T\left(i, j_{1}\right)+T\left(i, j_{2}\right)+i-2 .
$$

(For example, the bounding rectangle of the square configuration in Fig. 4 would contribute $T(3,5)$ "middle" parts, while the two resulting subrectangles after the horizontal cut is made would each contribute $T(1,5)$ "middle" parts.) We distinguish three cases:

Case 1: $j \leq 5$. Then

$$
T(i, j) \geq i-2 \geq \frac{(i-2)(j-2)}{3} .
$$

The last inequality is satisfied by the choice of $j$. 


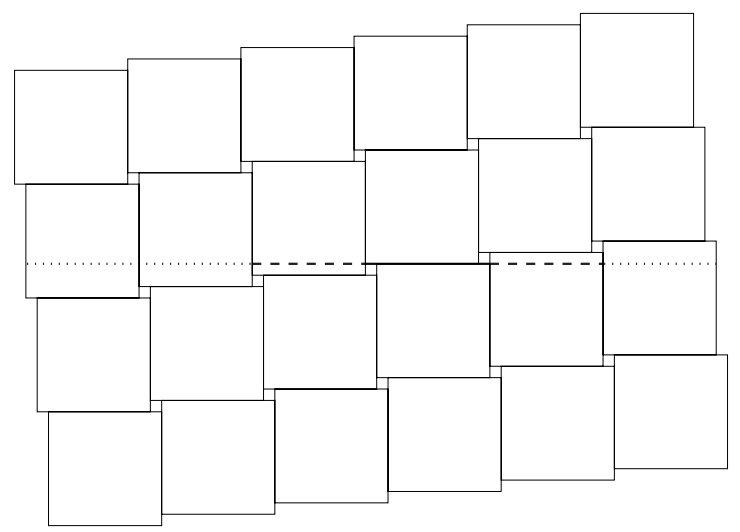

Fig. 4. A $4 \times 6$ square configuration (an instance for $T(3,5))$; the three portions of the cut are: border (solid), aligned (dashed) and middle (dotted).

Case 2: $j \geq 6$ and $j_{1} \leq 2$. Since $j_{2} \geq j-3 \geq 3$, we can use the inductive bound on $T\left(i, j_{2}\right)$ :

$$
\begin{aligned}
T(i, j) & \geq T\left(i, j_{2}\right)+i-2 \\
& \geq T(i, j-3)+i-2 \\
& \geq \frac{(i-2)(j-5)}{3}+i-2 \\
& =\frac{(i-2)(j-2)}{3} .
\end{aligned}
$$

Case 3: $j \geq 6$ and $j_{1}, j_{2} \geq 3$. Using the inductive bounds on both terms yields

$$
\begin{aligned}
T(i, j) & \geq T\left(i, j_{1}\right)+T\left(i, j_{2}\right)+i-2 \\
& \geq \frac{(i-2)\left(j_{1}-2\right)}{3}+\frac{(i-2)\left(j_{2}-2\right)}{3}+i-2 \\
& =\frac{(i-2)\left(j_{1}+j_{2}-1\right)}{3} \\
& \geq \frac{(i-2)(j-2)}{3} .
\end{aligned}
$$

This completes the proof of our claim.

We note that, most likely, the constant in the lower bound offered by our square configuration cannot be improved substantially, if at all; certainly, BSPs of size smaller than $2.5 n$ can be constructed for this configuration: e.g., using a $3 \times k$ strip cutting yields a BSP of size $\approx \frac{22}{9} k^{2} \approx 2.444 n$. 


\section{Segments in Higher Dimensions and Rectangles in $\mathbb{R}^{3}$}

\subsection{Segments in Three Dimensions}

Let $E=X \cup Y \cup Z$ be a set of $n$ axis-parallel segments in 3-space, where $X$ (resp. $Y, Z$ ) is the subset of segments of $E$ that are parallel to the $x$-axis (resp. to the $y$-axis, $z$-axis). Put $x=|X|, y=|Y|, z=|Z|$, so that $x+y+z=n$. For simplicity of presentation, suppose that the segments of $E$ are in general position. (Our arguments apply also in degenerate cases, but some notation becomes more complex.)

We construct a binary space partition of $E$ in the following simple manner.

(i) If one of $x, y, z$ is zero, say $z=0$, then we can obtain a BSP of size $O(n)$ by a sequence of horizontal cuts.

(ii) Suppose next that each of $x, y, z$ is at least one and that $y \leq x \leq z$. Put $t=$ $\lceil 2 \sqrt{x y / z}\rceil$. We partition space into a stack of $t$ slabs $\sigma_{1}, \ldots, \sigma_{t}$ by a sequence of cuts normal to the $z$-axis, so that at most $x / t$ segments in $X$ intersect the interior of any slab; this can be achieved by making each cutting plane contain one of the segments of $X$. Let $x_{i}, y_{i}, z_{i}$ respectively denote the number of segments in $X, Y, Z$ that intersect the interior of slab $\sigma_{i}$. We clearly also have $\sum_{i} y_{i} \leq y$.

For each slab $\sigma_{i}$, project all the segments of $E$ that intersect $\sigma_{i}$ onto the $x y$-plane. We obtain $x_{i}$ segments parallel to the $x$-axis, $y_{i}$ segments parallel to the $y$-axis, and $z_{i}$ points. We partition the segments into subsegments at their intersection points. The number of such points is $k_{i} \leq x_{i} y_{i}$ and the total number of subsegments is $x_{i}+y_{i}+2 k_{i} \leq$ $x_{i}+y_{i}+2 x_{i} y_{i}$.

We apply the planar binary space partitioning scheme of Theorem 2.1, and note that none of the $z_{i} \leq z$ singleton points will be split. We lift this planar partitioning scheme into three dimensions, lifting each cut by a line in the $x y$-plane to a cut by the plane containing the line and parallel to the $z$-axis. To complete the BSP within $\sigma_{i}$, we add a cut normal to the $x$-axis through each of the $z_{i} z$-parallel segments. It follows that the size of the partition within $\sigma_{i}$ is at most

$$
2\left(x_{i}+y_{i}+2 x_{i} y_{i}\right)+z_{i} \leq 2 x_{i}+2 y_{i}+4 y_{i} x / t+z .
$$

Hence the overall size of the BSP is at most

$$
2 x+2 y+4 x y / t+z t \leq 4 \sqrt{x y z}+2 x+2 y+2 z \leq 4 \sqrt{x y z}+2 n .
$$

We have thus shown:

Theorem 3.1. Let E be a collection of n segments in 3-space, consisting of x segments parallel to the $x$-axis, y segments parallel to the $y$-axis, and $z$ segments parallel to the z-axis. Then $E$ admits a BSP of size at most $4 \sqrt{x y z}+2 n$.

Remark. The maximum value of this bound is easily seen to be at most $(4 / 3 \sqrt{3}) n^{3 / 2}+$ $2 n$. This improves significantly the constant in the bound given by Paterson and Yao [10]. A lower bound construction due to Thurston [10] requires a BSP of size at least $(1 / 3 \sqrt{3})$ $n^{3 / 2}+n$. This leaves the open problem of tightening the gap of the factor 4 in the constant of proportionality between these bounds. 


\subsection{Segments in Higher Dimensions}

Let $E=X_{1} \cup X_{2} \cup \cdots \cup X_{d}$ be a set of $n$ axis-parallel segments in $d$-space, where $X_{i}$ is the subset of segments of $E$ that are parallel to the $x_{i}$-axis, for $i=1, \ldots, d$. Put $n_{i}=\left|X_{i}\right|$, for $i=1, \ldots, d$, so that $n_{1}+\cdots+n_{d}=n$.

We re-establish the following result of Paterson and Yao [10] with a simpler proof which also gives better constants of proportionality. As noted by Paterson and Yao [10], the upper bound is tight in the worst case-see also Section 4 below for an extended lower bound.

Theorem 3.2. Let $E$ be a collection of $n$ segments in $d$-space, for any $d \geq 3$, consisting of $n_{i}$ segments parallel to the $x_{i}$-axis, for each $i$. Then $E$ admits a BSP of size at most

$$
4^{1 /(d-1)}(d-1)\left(n_{1} n_{2} \cdots n_{d}\right)^{1 /(d-1)}+2 n
$$

Proof. We proceed by induction on $d$, where the base case $d=3$ has already been treated. We assume, for simplicity of presentation, that the segments of $E$ are in general position.

(i) If one of the $n_{i}$ 's is zero, say $n_{d}=0$, then we can obtain a BSP of linear size by a sequence of cuts orthogonal to the $x_{d}$-axis. Put

(ii) Suppose next that each of the $n_{i}$ 's is at least one and that $n_{d} \leq n_{d-1} \leq \cdots \leq n_{1}$.

$$
t=\left\lceil\frac{\left(4 n_{1} n_{2} \cdots n_{d-1}\right)^{1 /(d-1)}}{n_{d}^{(d-2) /(d-1)}}\right\rceil \geq n_{1}^{1 /(d-1)} \geq\left(\frac{n}{d}\right)^{1 /(d-1)}
$$

We partition space into a stack of $t$ slabs $\sigma_{1}, \ldots, \sigma_{t}$ by a sequence of cuts orthogonal to the $x_{d}$-axis, so that the following property holds. Let $n_{i}^{(\xi)}$ denote the number of segments in $X_{i}$ that intersect (the interior of) the slab $\sigma_{\xi}$. We require that $n_{1}^{(\xi)} \leq n_{1} / t$, for each $\xi$ (as in the three-dimensional case, this can be achieved by making each cutting hyperplane contain a segment of $X_{1}$ ). We clearly also have $\sum_{\xi} n_{i}^{(\xi)} \leq n_{i}$, for each $2 \leq i<d$.

For each slab $\sigma_{\xi}$, project all the segments of $E$ that cross $\sigma_{\xi}$ onto the hyperplane $x_{d}=0$. We obtain a collection of $n_{1}^{(\xi)}+\cdots+n_{d-1}^{(\xi)}$ segments which, by our general position assumption, are pairwise disjoint (since $d \geq 3$ ), and $n_{d}^{(\xi)}$ points. We apply the partitioning algorithm for $d-1$ dimensions, provided by the induction hypothesis, to the projected set, lifting, along the $x_{d}$ dimension, each $(d-2)$-dimensional cut performed by this algorithm to a $(d-1)$-dimensional cut (within $\left.\sigma_{\xi}\right)$. Note that the presence of points in the input has little effect on the algorithm and adds only a linear term to the size of the resulting BSP: we simply ignore the points and apply the algorithm only to the segments. When we are done, we take the cells of the resulting BSP that contain the input points, and split any such cell that contains more than one point into subcells, say by a sequence of parallel cuts. 
By the induction hypothesis, the size of the resulting BSP is at most

$$
\begin{gathered}
\sum_{\xi}\left[4^{1 /(d-2)}(d-2)\left(n_{1}^{(\xi)} n_{2}^{(\xi)} \cdots n_{d-1}^{(\xi)}\right)^{1 /(d-2)}+2\left(n_{1}^{(\xi)}+n_{2}^{(\xi)}+\cdots+n_{d-1}^{(\xi)}\right)+n_{d}^{(\xi)}\right] \\
\leq 4^{1 /(d-2)}(d-2)\left(\frac{n_{1}}{t}\right)^{1 /(d-2)} \cdot \sum_{\xi}\left(n_{2}^{(\xi)} \cdots n_{d-1}^{(\xi)}\right)^{1 /(d-2)} \\
\quad+2\left(n_{1}+n_{2}+\cdots+n_{d-1}\right)+t n_{d} .
\end{gathered}
$$

A multidimensional variant of Hölder's inequality easily implies that

$$
\sum_{\xi}\left(n_{2}^{(\xi)} \cdots n_{d-1}^{(\xi)}\right)^{1 /(d-2)} \leq\left(n_{2} \cdots n_{d-1}\right)^{1 /(d-2)} .
$$

Thus we conclude that the size of our BSP is at most

$$
\begin{gathered}
4^{1 /(d-2)}(d-2)\left(\frac{n_{1}}{t}\right)^{1 /(d-2)} \cdot\left(n_{2} \cdots n_{d-1}\right)^{1 /(d-2)}+2\left(n_{1}+n_{2}+\cdots+n_{d-1}\right)+t n_{d} \\
=4^{1 /(d-2)}(d-2)\left(\frac{n_{1} n_{2} \cdots n_{d-1}}{t}\right)^{1 /(d-2)} \\
\quad+2\left(n_{1}+n_{2}+\cdots+n_{d-1}\right)+t n_{d} .
\end{gathered}
$$

Our choice of $t$ implies that this is at most

$$
4^{1 /(d-1)}(d-1)\left(n_{1} n_{2} \cdots n_{d}\right)^{1 /(d-1)}+2\left(n_{1}+n_{2}+\cdots+n_{d}\right) .
$$

This establishes the induction step and thus completes the proof of the theorem.

Remark. Theorem 4.1 given below subsumes in general Theorems 3.1 and 3.2. We have considered these theorems separately because they also apply to situations where the sizes of the sets $X_{i}$ are unbalanced and because their more careful analysis leads to smaller constants of proportionality.

\subsection{Rectangles in Three Dimensions}

Let $\mathcal{R}$ be a set of $n$ pairwise disjoint axis-parallel rectangles in 3-space, and let $E$ denote the set of their edges. Write $E=X \cup Y \cup Z$, as above, and put $x=|X|, y=|Y|$, $z=|Z|$, so that $x+y+z=4 n$. We establish the following theorem; the upper bound $O\left(n^{3 / 2}\right)$ was also obtained by Paterson and Yao [10].

Theorem 3.3. Let $\mathcal{R}$ be a collection of $n$ axis-parallel rectangles in 3-space, having a total of $x$ edges parallel to the $x$-axis, $y$ edges parallel to the $y$-axis, and $z$ edges parallel to the $z$-axis, where $z \leq y \leq x$. Then $\mathcal{R}$ admits a BSP of size $O(n(\sqrt{z}+1))=O\left(n^{3 / 2}\right)$.

Proof. We construct a binary space partition of $\mathcal{R}$ in the following manner. We can assume without loss of generality that $z \neq 0$, since otherwise all rectangles are horizontal, and we can construct a linear-size BSP as in the previous proof. Since $z \leq y \leq x$, 
we have $z \leq 4 n / 3$ and $x+y \geq 8 n / 3$. Put

$$
t=\left\lceil\frac{x+y}{\sqrt{z}}\right\rceil \geq \frac{(8 n / 3)}{\sqrt{4 n / 3}}=\frac{4 \sqrt{3}}{3} \sqrt{n} .
$$

We partition space into $t$ horizontal slabs $\sigma_{1}, \ldots, \sigma_{t}$, as in the proof of Theorem 3.1, so that $x_{i}+y_{i} \leq\lceil(x+y) / t\rceil$, for each $i$, where $x_{i}, y_{i}, z_{i}$ are as defined in Section 3. We then have

$$
x_{i} y_{i} \leq\left(\frac{x_{i}+y_{i}}{2}\right)^{2} \leq \frac{1}{4}\left\lceil\frac{x+y}{t}\right\rceil^{2} \leq \frac{(\sqrt{z}+1)^{2}}{4} \leq z .
$$

For any $i$, let $R_{i}$ be the set of rectangles that intersect the open slab $\sigma_{i}$. These rectangles are of two kinds: black rectangles, which have a horizontal edge inside $\sigma_{i}$, and red rectangles, which do not. Note that red rectangles contribute to $z_{i}$ but not to $x_{i}$ and $y_{i}$; there are at most $z_{i} / 2 \leq z / 2$ red rectangles in $R_{i}$. Black rectangles are either horizontal rectangles fully contained in $\sigma_{i}$ or vertical rectangles that "start" and/or "end" within $\sigma_{i}$.

We project $\sigma$ onto the $x y$-plane. The projections of the red rectangles are red segments that are pairwise disjoint and are also disjoint from the projection of any black rectangle. Those black projections can be either segments or rectangles, and they can intersect (or overlap) each other.

Let $G$ be the (nonuniform) grid formed in the $x y$-plane by the horizontal and vertical lines that contain the edges of the projections of the black rectangles. We refer to the atomic rectangles of $G$ as pixels. We classify those pixels into red pixels, which intersect a red segment, and the remaining black pixels. There are a total of $O\left(x_{i} y_{i}\right)$ pixels. The black pixels can be grouped into black strips, which are maximal sets of consecutive black pixels within a single column of $G$ (i.e., pixels with a common $x$-projection).

We now apply the two-dimensional BSP construction (provided by Theorem 2.1) to the collection of black strips and red segments. We obtain a decomposition of the $x y$-plane into $O\left(x_{i} y_{i}+z_{i}\right)$ rectangular subregions. Moreover, any red segment or black strip is split by the algorithm at most once.

We lift the BSP just constructed in the $z$-direction, to obtain a partition of the slab $\sigma$ by vertical planes orthogonal to the $x$-and the $y$-axes. Let $K$ be a cell produced by this partitioning. If $K$ projects to a (portion of a) black strip, then it needs further partitioning, which we do as follows; refer to Fig. 5. Ignoring black rectangles that overlap the boundary of $K$ (which are not part of the subproblem at $K$ anyway), any other black rectangle that intersects $K$ crosses it from left to right, i.e., neither of its edges that are orthogonal to the $x$-axis meets $K$. Project $K$ onto the $y z$-plane. By the observation just made, the $n_{K}$ black rectangles that intersect $K$ project to a collection of $n_{K}$ pairwise disjoint segments, and we can again apply the two-dimensional BSP construction within this projection, effectively obtaining a BSP for $K$ that uses only cuts parallel to the $x$-axis, whose size is $O\left(n_{K}\right)$.

We claim that $\sum_{K} n_{K}=O\left(x_{i} y_{i}\right)$. Indeed, a black rectangle that is counted in $n_{K}$ must have an edge parallel to the $x$-axis that intersects $K$ (possibly overlapping with its boundary). This follows from the fact that any black rectangle that violates this property must be horizontal and its $x y$-projection must cover that of $K$ completely, as well as the pixels lying immediately above and below $K$ (in the $y$-direction). However, these pixels are red, by construction, so no horizontal black rectangle can cross them. This 


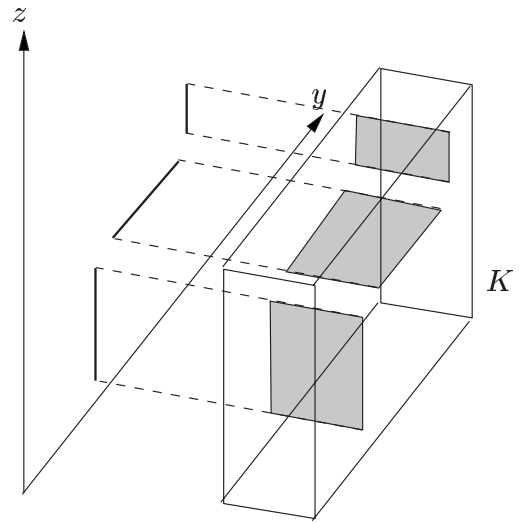

Fig. 5. A cell $K$ projecting onto a black strip, the black rectangles in $K$, and their projections onto the $y z$-plane.

contradiction establishes the asserted property. Now an ( $x$-parallel) edge of a black rectangle can cross at most $x_{i}$ black regions, and since we have only $y_{i}$ such edges, we conclude that $\sum_{K} n_{K}=O\left(x_{i} y_{i}\right)$.

We have thus constructed a BSP of size $O\left(x_{i} y_{i}+z_{i}\right)=O(z)$ for each of the $t$ slabs $\sigma_{i}$, thus obtaining an overall BSP of size $O(z t)=O\left((x+y) z^{1 / 2}\right)$. This completes the proof of the theorem.

\section{Arbitrary Hyperrectangles in Higher Dimensions}

Let $\mathcal{R}$ be a set of $n$ axis-parallel $k$-dimensional hyperrectangles ( $k$-rectangles, or just rectangles, in short) in $\mathbb{R}^{d}$. We assume that $k<d / 2$ and, for simplicity, that the $k$ rectangles are in general position. (Our arguments apply also to degenerate sets of $k$ rectangles.) Our assumptions imply that no two rectangles intersect.

Each rectangle $r \in \mathcal{R}$ has $k$ extent coordinates, i.e., coordinates $x_{i}$ for which the projection of $r$ onto the $x_{i}$-axis is an interval with nonempty interior, and $d-k$ fixed coordinates (those for which this projection is a singleton point).

Let $K$ be an axis-parallel box in $\mathbb{R}^{d}$, and let $r$ be a rectangle in $\mathcal{R}$. We say that $r$ stabs $K$ in the $x_{i}$-direction, or that $r$ is an $i$-stabber of $K$, if $r$ intersects both facets of $K$ normal to the $x_{i}$-axis. Let $\operatorname{stab}(r, K) \subseteq\{1,2, \ldots, d\}$ denote the set of coordinate directions in which $r$ stabs $K$. We say that $r$ is an $M$-stabber of $K$ if $\operatorname{stab}(r, K)=M$. If $|M|=q$, we say that $r$ stabs $K$ q times.

The main result of this section is given in the following two theorems:

Theorem 4.1. For any $n, d$, and $k<d / 2$, any set of $n$ axis-parallel $k$-rectangles in $d$-space admits a BSP of size $O\left(n^{d /(d-k)}\right)$.

Theorem 4.2. For any $n, d$, and $k<d / 2$, there is a set of $n$ axis-parallel $k$-rectangles in $d$-space such that any rectilinear BSP of it has size $\Omega\left(n^{d /(d-k)}\right)$. 
Proof of Theorem 4.1. Let $\mathcal{R}$ be a set of $n k$-rectangles in $d$-space, for some $n, d$, and $k<d / 2$. Put $t=c n^{1 /(d-k)}$, for some absolute constant $c>1$. The BSP construction proceeds through $d$ phases, where in the $j$ th phase we cut each of the cells produced in the preceding phases by hyperplanes orthogonal to the $x_{j}$-axis. Each cut that we perform is at some fixed coordinate of some rectangle in $\mathcal{R}$.

In the first phase we slice $\mathbb{R}^{d}$ by a sequence of $t-1$ hyperplanes orthogonal to the $x_{1}$-axis, partitioning space into $t$ slabs, so that each slab $\sigma$ contains at most $n / t$ rectangles that are either orthogonal to $x_{1}$ or have an extent in the $x_{1}$-coordinate but are not 1-stabbers of $\sigma$.

Suppose we are about to start the $j$ th phase. Let $\sigma$ be a cell (subslab) produced by the previous phases. We inductively assume the following size invariant: for any subset $M \subseteq\{1,2, \ldots, j-1\}$ of size $|M| \leq k$, the slab $\sigma$ contains at most $n / t^{j-1-|M|}$ $M$-stabbers. The size invariant holds trivially when $j=2$.

We cut $\sigma$ by $O(t)$ cuts orthogonal to the $x_{j}$-axis, to ensure that, for each subset $M$ as above, any resulting subslab $\sigma^{\prime}$ contains at most $n / t^{j-|M|} M$-stabbers of $\sigma$ that are not $j$-stabbers of $\sigma^{\prime}$. (By the induction hypothesis, $\sigma$ contains at most $n / t^{j-1-|M|}$ such rectangles, so it is easy to cut this number down by a factor of $t$ for each $\sigma^{\prime}$.) In addition, for each subset $M$ of $\{1, \ldots, j\}$ of size at most $k$ that contains $j, \sigma^{\prime}$ contains at most $n / t^{j-|M|} M$-stabbers. (These bounds are simply "carried over" from the inductively assumed bounds for $\sigma$ and $j-1$.) This establishes the size invariant for the next phase.

Let us analyze the performance of this partitioning scheme. We claim that, since $c>1$, none of the final cells can have any rectangle meeting its interior. Indeed, let $\sigma$ be a final cell. By the size invariant, for each set $M$ of coordinates of size $|M| \leq k, \sigma$ has at most

$$
\frac{n}{t^{d-|M|}}=\frac{1}{c^{d-|M|} n^{((d-|M|) /(d-k))-1}}=\frac{1}{c^{d-|M|} n^{(k-|M|) /(d-k)}} \leq \frac{1}{c^{d-k}}
$$

$M$-stabbers. By choosing $c>1$ we are guaranteed that the interior of $\sigma$ does not meet any rectangle of $\mathcal{R}$.

Hence the resulting subdivision is indeed a BSP for $\mathcal{R}$. The number of cells of this BSP is clearly

$$
O\left(\left(\begin{array}{l}
d \\
k
\end{array}\right) t^{d}\right)=O\left(\left(\begin{array}{l}
d \\
k
\end{array}\right) c^{d} n^{d /(d-k)}\right)=O\left(n^{d /(d-k)}\right)
$$

the constant of proportionality thus depends exponentially on $d$ and $k$. Further, any one rectangle is cut into at most $t^{k}=n^{k /(d-k)}$ pieces, implying a bound of $O\left(n^{d /(d-k)}\right)$ on the number of fragments. This completes the proof of the theorem.

Proof of Theorem 4.2. Our construction generalizes the construction of Thurston (reported by Paterson and Yao [10]) that implies the $\Omega\left(n^{3 / 2}\right)$ lower bound for disjoint 2-rectangles in 3-space. Put $I=\left[0, n^{1 /(d-k)}+1\right]$, and let $K$ be the cube $I^{d}$. Put $L=\left(\begin{array}{l}d \\ k\end{array}\right)$. For each set $M$ of $k$ coordinates, we construct $n k$-rectangles whose extent coordinates are those of $M$, as follows. Put $E=\left\{1,2, \ldots, n^{1 /(d-k)}\right\}^{d-k}$. For each $\mathbf{a} \in E$ construct a rectangle $r=r(\mathbf{a})$ whose $i$ th fixed coordinate (i.e., the $i$ th smallest among those coordinates not in $M)$ is $\mathbf{a}_{i}+\varepsilon(\mathbf{a}, M)$, and whose projection on each of the extent co- 
ordinates (i.e., those in $M)$ is $I$. Here $\varepsilon(\mathbf{a}, M)$ is a number in $(0,1)$, so that different pairs $(\mathbf{a}, M)$ are assigned different numbers. We thus obtain a collection $\mathcal{R}$ of a total of $L n$ rectangles.

We claim that any axis-parallel BSP for $\mathcal{R}$ must consist of $\Omega\left(n^{d /(d-k)}\right)$ cells. Consider the integer grid $G=\left\{1,2, \ldots, n^{1 /(d-k)}\right\}^{d}$. With each $\mathbf{g} \in G$ associate the grid cell $Q(\mathbf{g})=\prod_{i=1}^{d}\left(\mathbf{g}_{i}, \mathbf{g}_{i}+1\right)$. A grid cell $Q=Q(\mathbf{g})$ is crossed by exactly $L$ rectangles of $\mathcal{R}$ : for each $k$-tuple of coordinates there is exactly one rectangle of $\mathcal{R}$ whose extent coordinates are those in the tuple, that crosses $Q$-it is the rectangle whose fixed coordinates agree (up to the increments $\varepsilon(\mathbf{a}, M)$ ) with the corresponding elements of $\mathbf{g}$.

Since $L>1$, any BSP for $\mathcal{R}$ must have cuts that cross $Q$, since otherwise $Q$ would be contained in a single cell of the BSP, which is impossible since that cell would be crossed by more than one rectangle of $\mathcal{R}$. Moreover, we argue that $Q$ must be cut by (portions of) hyperplanes in at least $k$ different orientations. (We omit the easy proof.) Halt the BSP construction immediately after $Q$ is cut for the first time by hyperplanes in $k$ different orientations; suppose that they are the first $k$ coordinates. Let $r$ be the unique rectangle of $\mathcal{R}$ that crosses $Q$ and whose extent coordinates are the first $k$ coordinates. For each $i=1, \ldots, k$ let $h_{i}$ be a hyperplane orthogonal to $x_{i}$ that has already crossed $Q$. Then $r \cap\left(\cap_{i=1}^{k} h_{i}\right)$ is a singleton point $v$-a vertex of a portion of $r$ that the BSP has just formed. We assign $Q$ to this portion of $r$, or, more precisely, to the final portion of $r$ that will be formed by the BSP and will have $v$ as a vertex. No such portion can be charged by more than $2^{k}$ grid cells, which implies that the number of fragments of rectangles in $\mathcal{R}$ that the BSP has to form is at least proportional to the number of grid cells, so the BSP has size $\Omega\left(n^{d /(d-k)}\right)$, as asserted.

Remark. Theorem 4.1 holds also for any integer $k$ satisfying $d / 2 \leq k \leq d-1$, even if the rectangles in $\mathcal{R}$ are allowed to intersect. The lower bound applies also for any integer $k$ satisfying $d / 2 \leq k \leq d-1$, but the construction uses rectangles that intersect. The proofs are essentially identical.

\section{Disjoint 2-Rectangles in $\mathbb{R}^{4}$}

In this section we consider BSPs for sets of $n$ axis-parallel pairwise disjoint 2-rectangles in $\mathbb{R}^{4}$. This is the simplest instance not covered by Theorems 4.1 and 4.2.

Theorem 5.1. For any $n$, any set of $n$ axis-parallel pairwise disjoint 2-rectangles in $\mathbb{R}^{4}$ admits a rectilinear $B S P$ of size $O\left(n^{5 / 3}\right)$.

Theorem 5.2. For any $n$, there is a set of $n$ axis-parallel pairwise disjoint 2-rectangles in $\mathbb{R}^{4}$ such that any rectilinear BSP of it has size $\Omega\left(n^{5 / 3}\right)$.

Proof of Theorem 5.1. Let $\mathcal{R}$ be any set of $n$ axis-parallel pairwise disjoint 2-rectangles in $\mathbb{R}^{4}$. Let $K$ be an axis-parallel box in $\mathbb{R}^{4}$. Apply the same round-robin construction given in the preceding section, but with $t=c^{\prime} n^{1 / 6}$ for an appropriate constant $c^{\prime}$. We obtain $O\left(n^{2 / 3}\right)$ subcells, so that each subcell $\sigma$ contains at most $n / t^{2}=O\left(n^{2 / 3}\right)$ rectangles 
that stab $\sigma$ twice, at most $n / t^{3}=O\left(n^{1 / 2}\right)$ rectangles that stab $\sigma$ once, and at most $n / t^{4}=O\left(n^{1 / 3}\right)$ rectangles that do not stab $\sigma$ at all.

Lemma 5.3. No two rectangles can stab a subcell $\sigma$ in complementary sets of coordinate directions.

Proof. Any two such rectangles must intersect, contrary to assumption.

By Lemma 5.3, it is easily verified that there are only two possible maximal values for the set $S_{2}(\sigma)=\{\{i, j\} \mid \sigma$ has an $\{i, j\}$-stabber $\}$, up to a permutation of the coordinates; namely:

(i) $S_{2}(\sigma) \subseteq\{(1,2),(1,3),(2,3)\}$,

(ii) $S_{2}(\sigma) \subseteq\{(1,4),(2,4),(3,4)\}$.

Case (i). Suppose all the rectangles that stab $\sigma$ once are orthogonal to the $x_{4}$-axis and lie at different heights. We cut $\sigma$ by $O\left(n^{1 / 6}\right)$ cuts orthogonal to the $x_{4}$-axis so that each of the $O\left(n^{5 / 6}\right)$ subcells, resulting from all cells $\sigma$, contains at most $n^{1 / 2}$ (portions of) rectangles. In particular, each subcell $\sigma^{\prime}$ contains at most $n^{1 / 2}$ rectangles that stab $\sigma^{\prime}$ twice, at most $n^{1 / 3}$ rectangles that stab $\sigma^{\prime}$ once, and at most $n^{1 / 6}$ rectangles that do not stab $\sigma^{\prime}$ at all. By Lemma 5.3, the extent coordinates of the double stabbers of a subcell $\sigma^{\prime}$ will again fall into either case (i) or case (ii) (with a possible new permutation of the coordinates). For a subcase (i) subcell $\sigma^{\prime}$, we cut $\sigma^{\prime}$ by $O\left(n^{1 / 6}\right)$ cuts orthogonal to the $x_{4}$ axis, resulting in a total of $O(n)$ sub-subcells (over all original cells $\sigma$ ) each containing at most $n^{1 / 3}$ (portions of) rectangles. For a subcase (ii) subcell $\sigma^{\prime}$, we apply the first part of the case (ii) decomposition, described below, which consists of a round-robin cutting of each subcell into $\left(n^{1 / 6}\right)^{3}=n^{1 / 2}$ pieces each containing at most $n^{1 / 6}$ (portions of) rectangles. If we denote by $F(n)$ the maximum size of a BSP that the algorithm constructs for any input set of $n$ pairwise disjoint axis-parallel 2-rectangles in $\mathbb{R}^{4}$, then the overall number of final cells produced for cells $\sigma$ that belong to case (i) is $O(n) F\left(n^{1 / 3}\right)+O\left(n^{4 / 3}\right) F\left(n^{1 / 6}\right)$.

Case (ii). Now suppose all the rectangles that stab $\sigma$ twice are parallel to the $x_{4}$-axis. We first execute a round-robin procedure that only makes cuts orthogonal to the $x_{1^{-}}, x_{2^{-}}$, and $x_{3}$-axes. At each stage of this procedure we make $t=O\left(n^{1 / 6}\right)$ cuts. This partitions each of the preceding $O\left(n^{2 / 3}\right)$ cells into $O\left(n^{1 / 2}\right)$ subcells, for a total of $O\left(n^{7 / 6}\right)$ subcells. This can be done so that each subcell $\sigma$ contains

- at most $n^{2 / 3} / t^{2}=n^{1 / 3}$ rectangles that stab $\sigma$ in the $x_{4}$-direction and one other direction,

- at most $n^{1 / 2} / t=n^{1 / 3}$ rectangles that stab $\sigma$ twice, but not in the $x_{4}$-direction,

- at most $n^{1 / 2} / t^{2}=n^{1 / 6}$ rectangles that stab $\sigma$ once,

- and no other rectangles.

(Note that when this decomposition is applied to the relevant subcells arising in the preceding case (i) decomposition, the numbers of various rectangles within each subcell are smaller, thereby resulting in output subcells with fewer rectangles per subcell.)

We next refine each subcell further. Lemma 5.3 implies that the new double stabbers of $\sigma$ fall into either case (i) or case (ii) (with a possible new permutation of the coordinates). 
In subcase (i) we proceed in a manner similar to the one above, cutting $\sigma$ by $O\left(n^{1 / 6}\right)$ cuts orthogonal to the $x_{4}$-axis, to obtain $O\left(n^{1 / 6}\right)$ subcells, each containing at most $n^{1 / 6}$ rectangles, for an overall recursive bound of the form $O\left(n^{4 / 3}\right) \cdot F\left(n^{1 / 6}\right)$.

In subcase (ii) we again proceed as above, cutting $\sigma$ in a round-robin fashion by cuts orthogonal to the $x_{1^{-}}, x_{2^{-}}$, and $x_{3}$-axes, making $t=O\left(n^{1 / 6}\right)$ cuts in each round. This can be done to eliminate the at most $n^{1 / 6}$ rectangles that stab the subcell once, as well as all of the at most $n^{1 / 3}$ double stabbers. Hence this step produces a BSP for $\sigma$, whose size is $O\left(n^{1 / 2}\right)$, for a total of $O\left(n^{7 / 6} \cdot n^{1 / 2}\right)=O\left(n^{5 / 3}\right)$.

Putting everything together, we obtain the recurrence

$$
F(n)=O\left(n^{5 / 3}\right)+O(n) \cdot F\left(n^{1 / 3}\right)+O\left(n^{4 / 3}\right) \cdot F\left(n^{1 / 6}\right),
$$

whose solution is easily seen to be $F(n)=O\left(n^{5 / 3}\right)$.

Proof of Theorem 5.2. Let $G$ be the $n^{2 / 3} \times n^{1 / 3} \times n^{1 / 3} \times n^{1 / 3}$ integer grid in 4-space. Let $I$ denote the interval $\left[0, n^{1 / 3}+1\right]$ and let $I^{\prime}$ denote the interval $\left[0, n^{2 / 3}+1\right]$. We start with the following four families of "prototype" rectangles:

$$
\begin{aligned}
& \mathcal{R}_{1}=\left\{\{i\} \times\{j\} \times I \times I \mid 1 \leq i \leq n^{2 / 3}, 1 \leq j \leq n^{1 / 3}\right\}, \\
& \mathcal{R}_{2}=\left\{\{i\} \times I \times\{k\} \times I \mid 1 \leq i \leq n^{2 / 3}, 1 \leq k \leq n^{1 / 3}\right\}, \\
& \mathcal{R}_{3}=\left\{\{i\} \times I \times I \times\{l\} \mid 1 \leq i \leq n^{2 / 3}, 1 \leq l \leq n^{1 / 3}\right\}, \\
& \mathcal{R}_{4}=\left\{I^{\prime} \times[j, j+\varepsilon] \times[k, k+\varepsilon] \times[l, l+\varepsilon] \mid 1 \leq j, k, l \leq n^{1 / 3}\right\},
\end{aligned}
$$

where $\varepsilon=2^{-n}$. (The elements of $\mathcal{R}_{4}$ are 4-rectangles, but we can replace each one with its 24 two-dimensional faces.) We perturb the prototype rectangles by translating each one along a unique vector, with all coordinates less than $1 / n$, chosen so that no two rectangles intersect and all defining coordinates are distinct. The result is a set $\mathcal{R}=\mathcal{R}_{1} \cup \mathcal{R}_{2} \cup \mathcal{R}_{3} \cup \mathcal{R}_{4}$ of $O(n)$ pairwise disjoint rectangles in general position.

Fix any $1 \leq i \leq n^{2 / 3}, 1 \leq j, k, \ell \leq n^{1 / 3}$, and consider the cube $\Xi(i, j, k, \ell)$ of width 0.1 centered at $(i, j, k, l)$. We refer to $\Xi(i, j, k, \ell)$ as the junction at $(i, j, k, \ell)$. Each junction $\Xi(i, j, k, \ell)$ intersects exactly one rectangle $r_{m}=r_{m}(i, j, k, l)$ from each set $\mathcal{R}_{m}$.

Consider any rectilinear BSP for $\mathcal{R}$, namely, one that only uses cuts orthogonal to the coordinate axes. It is clear that each junction $\Xi=\Xi(i, j, k, \ell)$ must be cut by at least one hyperplane of the BSP, or else the BSP will have a final cell that intersects more than one rectangle of $\mathcal{R}$.

Claim 5.4. For each junction $\Xi=\Xi(i, j, k, \ell)$, any rectilinear BSP splits at least one of the rectangles $r_{1}, r_{2}, r_{3}$, and $r_{4}$ that intersect $\Xi$ into subrectangles with at least one vertex inside $\Xi$.

Intuitively, the only way to "get rid" of the box of $\mathcal{R}_{4}$ that cuts $\Xi$ by cuts parallel to $x_{1}$ is to cut along each of its three-dimensional facets; moreover, one cannot get rid of the other three rectangles in $\mathcal{R}_{1} \cup \mathcal{R}_{2} \cup \mathcal{R}_{3}$ that cross $\Xi$ without making at least two differently oriented cuts, each being orthogonal to one of the axes $x_{2}, x_{3}, x_{4}$. 
Proof of the Claim. Since $\Xi$ intersects four rectangles, it must be cut at least once. Let $h$ be the first such cut. If $h$ is normal to the $x_{1}$-axis, it splits $r_{4}$ into two fragments with a common vertex (in fact, eight common vertices) in $\Xi$. Otherwise, suppose $h$ is normal to the $x_{2}$-axis. Of the four rectangles intersecting $\Xi$, only $r_{1}$ can be eliminated by this cut. At least one of the two subcells created by $h$, say $\Xi^{\prime}$, intersects all three rectangles $r_{2}, r_{3}$, and $r_{4}$.

Since $\Xi^{\prime}$ intersects at least three rectangles, it must be cut at least once. Let $h^{\prime}$ be the first hyperplane to cut $\Xi^{\prime}$. As before, if $h^{\prime}$ is normal to the $x_{1}$-axis, then $h^{\prime} \cap r_{4}$ contains at least one fragment vertex inside $\Xi$. If $h^{\prime}$ is normal to the $x_{2}$-axis, then at least one of the two subcells of $\Xi^{\prime}$, say $\Xi^{\prime \prime}$, intersects all three rectangles $r_{2}, r_{3}$, and $r_{4}$. In this case we can argue recursively, using $\Xi^{\prime \prime}$ in place of $\Xi^{\prime}$ and $h^{\prime}$ in place of $h$. If $h^{\prime}$ is normal to the $x_{3}$-axis, then $h \cap h^{\prime} \cap r_{3}$ is a BSP vertex inside $\Xi$.

All other cases follow from symmetric arguments.

Each rectangle fragment has four vertices, and by our claim, each junction contains at least two fragment vertices. Thus, since there are $n^{5 / 3}$ junctions, the number of fragments is at least $n^{5 / 3} / 2$.

\section{Conclusion}

In conclusion, we refer again to our summary of results in Table 1. Perhaps the most interesting remaining open problem is to generalize our techniques for (disjoint) 2rectangles in $\mathbb{R}^{4}$ to higher dimensions, with the hope of obtaining asymptotically tight bounds for $k$-rectangles in $\mathbb{R}^{d}$, for all $k$ and $d$.

\section{Acknowledgments}

We thank Shakhar Smorodinsky and Saurabh Sethia for several useful discussions and valuable input on this work. We also thank the referees for their careful reading and valuable and detailed suggestions for improving the presentation.

\section{References}

1. F. d'Amore and P. G. Franciosa, On the optimal binary plane partition for sets of isothetic rectangles, Inform. Process. Lett. 44 (1992), 255-259.

2. M. de Berg, M. de Groot, and M. Overmars, New results on binary space partitions in the plane, Comput. Geom. Theory Appl. 8 (1997), 317-333.

3. M. de Berg, M. de Groot, and M. Overmars, Perfect binary space partitions, Comput. Geom. Theory Appl. 7 (1997), 81-91.

4. P. Berman, B. DasGupta, and S. Muthukrishnan, On the exact size of the binary space partitioning of sets of isothetic rectangles with applications, SIAM J. Discrete Math. 15(2) (2002), 252-267.

5. P. Berman, B. DasGupta, S. Muthukrishnan, and S. Ramaswami, Efficient approximation algorithms for tiling and packing problems with rectangles, J. Algorithms 41 (2001), 443-470.

6. S. Chin and S. Feiner, Near real-time shadow generation using BSP trees, Comput. Graph. 23 (1989), 99-106. 
7. S. E. Dorwald, A survey of object-space hidden surface removal, Internat. J. Comput. Geom. Appl. 4 (1994), 325-362.

8. H. Fuchs, Z. M. Kedem, and B. Naylor, On visible surface generation by a priori tree structures, Comput. Graph. 14 (1980), 124-133.

9. M. S. Paterson and F. F. Yao, Efficient binary space partitions for hidden-surface removal and solid modeling, Discrete Comput. Geom. 5 (1990), 485-503.

10. M. S. Paterson and F. F. Yao, Optimal binary space partitions for orthogonal objects, J. Algorithms $\mathbf{1 3}$ (1992), 99-113.

11. C. D. Tóth, A note on binary plane partitions, Discrete Comput. Geom. 30(1) (2003), 3-16.

12. C. D. Tóth, Binary space partitions for line segments with a limited number of directions, SIAM J. Comput. 32(2) (2003), 307-325.

13. W. C. Thibault and B. F. Naylor, Set operations on polyhedra using binary space partitioning trees, Comput. Graph. 21(4) (1987), 153-162 (Proc. SIGGRAPH '87).

14. G. Vaněček Jr., Brep-Index: a multidimensional space partitioning tree, Internat. J. Comput. Geom. Appl. 1(3) (1991), 243-261.

Received June 1, 2001, and in revised form August 20, 2002, and July 22, 2003.

Online publication December 19, 2003. 